\title{
Antitumor effect of recombinant Mycobacterium smegmatis expressing MAGEA3 and SSX2 fusion proteins
}

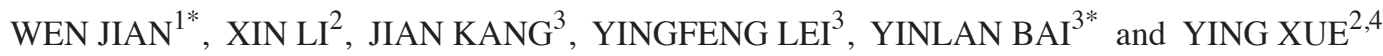 \\ ${ }^{1}$ Department of Respiratory Medicine, The First Affiliated Hospital of The Fourth Military Medical University, \\ Xi'an, Shaanxi 710038; ${ }^{2}$ Medical Oncology Center, Dongguan Kanghua Hospital, Dongguan, \\ Guangdong 523080; ${ }^{3}$ Department of Basic Microbiology, The Fourth Military Medical University; \\ ${ }^{4}$ Department of Radiation Oncology, The First Affiliated Hospital of The Fourth Military Medical University, \\ Xi'an, Shaanxi 710038, P.R. China
}

Received July 5, 2017; Accepted June 8, 2018

DOI: 10.3892/etm.2018.6425

\begin{abstract}
Mycobacterium smegmatis (M. smegmatis), which is a nonpathogenic and fast-growing mycobacterium, is a potential vaccine vector capable of expressing heterologous antigens. Spontaneous humoral and cellular immune responses have been demonstrated against cancer/testis antigens (CTA), including melanoma-associated antigen A (MAGEA) and SSX. In the present study, recombinant plasmids expressing MAGEA3 and SSX2 were constructed. The recombinant plasmids were transferred into $M$. smegmatis to generate the novel antitumor DNA vaccine. As MAGEA3 and SSX2 were in different ligation sequences, the two DNA vaccines were recombinant $M$. smegmatis MAGEA3-SSX2 (rM.S-MS) and recombinant $M$. smegmatis SSX2-MAGEA3 (rM.S-SM), respectively. The expression levels of Fusion proteins were assessed by western blotting. BALB/c mice were immunized with rM.S and western blot analysis was used to determine whether antibodies against MAGEA3 or SSX2 were produced in immunized mice. EC9706 cells were inoculated into $\mathrm{BALB} / \mathrm{c}$ nude mice and the mice were maintained until an obvious visible tumor appeared on the back. Subsequently, the blood from the rM.S immunized BALB/c mice was injected into the $\mathrm{BALB} / \mathrm{c}$ nude mice via the tail vein. In order to evaluate the antitumor effect of the vaccines, tumor volume and weight were measured 5 to 21 days after injection. Mice were euthanized on day 21 of tumor growth, and the tumor was dissected and weighed. The two fusion proteins were expressed in the rM.S and the specific fusion protein antibodies
\end{abstract}

Correspondence to: Dr Ying Xue, Medical Oncology Center, Dongguan Kanghua Hospital, Tuanjie 16 South Road, Dongguan, Guangdong 523080, P.R. China

E-mail: yingxuegx@163.com

${ }^{*}$ Contributed equally

Key words: DNA vaccine, MAGEA3, SSX2, tumor, Mycobacterium smegmatis were expressed in the blood of immunized BALB/c mice. The tumor volumes and weight in the recombinant $M$. smegmatis MAGEA3 (rM.S-M) and recombinant M. smegmatis SSX2 (rM.S-S) groups were significantly reduced compared with the control group. Furthermore, the decrease in tumor volumes and weight in the rM.S-MS and rM.S-SM groups was more severe than in the rM.S-M or rM.S-S groups. There was no significant difference in the antitumor effect of the rM.S-MS and rM.S-SM groups. The present findings suggest that this rM.S may be a potential candidate therapeutic vaccine for the treatment of cancer.

\section{Introduction}

Immunotherapy is a new avenue of cancer treatment for a range of different cancer types. It is now understood that the immune system is capable of recognizing and eliminating cancer cells, but tumors evade and suppress host immune responses and therefore persist and spread (1-3). During the past few decades, anticancer immunotherapy has evolved from a promising therapeutic option to a robust clinical reality. Many immunotherapeutic regimens are now approved for use in cancer patients, and many others are being investigated as standalone therapeutic interventions or combined with conventional treatments in clinical studies.

Mycobacterium smegmatis ( $M$. smegmatis) is a fast-growing saprophytic environmental bacterium, which is a non-pathogenic and commensal genus $(4,5)$. M. smegmatis also has a number of properties such as growth rapidily and can be transformed effectively with many genes, that renders it an ideal vaccine vector. Further more, M. smegmatis is reported to activate dendritic cells and trigger CD8-mediated immune responses, and immunization with rM.S can generate more durable memory $\mathrm{T}$ cells than intramuscular DNA vaccination $(6,7)$. These findings indicate the potential role of mycobacteria as recombinant vaccine delivery vector.

Immunogenic target antigen is another crucial element for developing a successful vaccine. The melanoma-associated antigen A3 (MAGEA3) is a member of the large cancer/testis antigens (CTA), which are frequently aberrantly expressed in a wide range of cancer (8-12). MAGEA gene family is regarded 
as a promising target of specific immunotherapy because MAGEA is expressed mainly in cancers that have acquired maliganat phenotypes and contribute towards malignancy (13). MAGEA3 is an tumor antigenic nonapeptide that is identified in various tumors and associated with a broad set of HLA (human MHC locus) molecules (14). Consequently, MAGEA3 antigen is a genuinely selective target for tumor-specific active immunotherapy.

It is well known that novel and effective adjuvants can elicit stronger cellular and humoral adaptive immune responses to antigenic targets. The expression of a particular CTA is limited to only a subset of patients with a particular tumor type; therefore, for human application, this is too weak to induce a substantial response against difficult antigens. In order to expand the number of patients and tumor types that can be treated, it is necessary to expand the repertoire of antigens by this approach. We developed another CTA, SSX2 (synovial sarcoma $\mathrm{X}$ breakpoint 2), which is the primary member of the SSX family expressed in different kinds of cancers inculding prostate, lung, breast and multiple myeloma and pancreatic cancer (15-19). SSX2 gene encodes for the human tumor-specific antigen HOM-MEL-40, which is an immunogenic protein known to trigger spontaneous antibody responses (20). The SSX2 protein can induce spontaneous immune responses. Therefore, the development of vectors expressing SSX2 opens up a wide array of possibilities in the immunotherapy of cancer.

In this study, we designed two fusion proteins with different ligation sequences, MAGEA3-SSX2 and SSX2-MAGEA3, from M. smegmatis for tumor immunotherapy and detected their tumor therapeutic effect by mice tumor-burdened experiments.

\section{Materials and methods}

Bacterial strains and growth conditions. The $M$. smegmatis strain MC $^{2} 155$ was supplied by Yinlan Bo's Laboratory at the Fourth Military Medical University (Xi'an, China). M. smegmatis cultures were grown in $7 \mathrm{H} 10$ solid medium (7H10 solid medium contained $3 \mathrm{ml} / 1$ glycerin, $0.5 \mathrm{~g} / \mathrm{l}$ Tween-80, $100 \mathrm{ml} / 1$ OADC and 19/1 middle brook 7H10 agar powder) and incubated at $37^{\circ} \mathrm{C}$ for 2-3 days; the medium was supplemented with hygromycin $(50 \mathrm{ng} / \mathrm{ml})$ when selecting for the recombinant plasmid. Escherichia coli cultures were grown in Luriae-Bertani (LB) broth or plates (LB broth contained $10 \mathrm{~g} / \mathrm{l}$ trypeptone; $15 \mathrm{~g} / \mathrm{l} \mathrm{NaCl} ; 5 \mathrm{~g} / 1$ yeast extract; LB plates contained $10 \mathrm{~g} / 1$ trypeptone; $15 \mathrm{~g} / 1 \mathrm{NaCl} ; 5 \mathrm{~g} / 1$ yeast extract and $15 \mathrm{~g} / \mathrm{l}$ agar powder) and incubated at $37^{\circ} \mathrm{C}$ overnight; the media were supplemented with ampicillin $(100 \mu \mathrm{g} / \mathrm{ml})$ when selecting for the recombinant plasmid.

Plasmid and strain construction. The pDE22 vector was supplied by Yinlan Bo's laboratory at the Fourth Military Medical University. The E. coli strain DH5- $\alpha$ was purchased from MBI Fermentas (Vilnius, Lithuania). The pUC57 vector was purchased from Tiangen (Beijing, China). Taq DNA polymerase and Pst I endonuclease were obtained from Takara Biotechnology Co., Ltd. (Dalian, China). BamHI endonuclease, ClaI endonuclease, EcoRV endonuclease and T4 DNA ligase were obtained from MBI Fermentas (Burlington,
ON, Canada). All other media components and chemicals used were of the highest purity grade available commercially from Beijing Chemical Plant, China.

Splicing overlap extension polymerase chain reaction (SOE-PCR) primers were synthesized by Shanghai Bioengineering Company (Shanghai, China). The MAGEA3 gene was cloned from DNA of EC9706 cell via PCR using the primer pair: Sense primer 5'-GCCGATATCATGCCT CTTGAGCAGAGGAGTC-3' and antisense primer 5'-GCT GCCGCCGCCGCCGCTGCC-3'. The SSX2 gene was cloned from DNA of EC9706 cell via PCR using the primer pair: Sense primer 5'-GCCGATATCATGAACGGAGAC GACGCCTTTC-3' and antisense primer 5'-GCTGCCGCC GCCGCCGCTGCC-3'. The cloned genes MAGEA3 and SSX2 were constructed from two kinds of different connection sequence gene fragments, MAGEA3-SSX2 (MS) and SSX2-MAGEA3 (SM). The MAGEA3-SSX2 fragment was amplified using the primer pair: Sense primer 5'-CGGCGG CGGCGGCAGCATGCCTCTTGAGCAGAG-3' and antisense primer 5'-CCATCGATTTACTCGTCATCTTCCTCA GGG-3', and the SSX2-MAGEA3 fragment was amplified using the primer pair: Sense primer 5'-CGGCGGCGGCGG CAGCATGAACGGAGACGACG-3' and antisense primer 5'-CCATCGATTCACTCTTCCCCCTCTCTCAAA-3'. The MAGEA3-SSX2 and SSX2-MAGEA3 fusion expression cassettes were generated using the gap repair method as above, and a linker designed was used to maintain the correct biological activity of both MAGEA3 and SSX2. A verified clone with the correct sequence (AuGCT Biotechnology, Beijing, China) was transferred into a pDE22 cloning vector, then cut with the appropriate restriction endonucleases and inserted in the E. coli-mycobacterium shuttle plasmid pDE22 construct. Plasmid DNA was introduced into M. smegmatis by electroporation using standard techniques (21) to generate the rM.S strain expressing the two kinds of fusion protein MAGEA3-SSX2 and SSX2-MAGEA3.

Western blot analysis. To monitor the expression of the M. smegmatis MAGEA3 and SSX2 transgenes, the rMS strains were grown in 7H10/ADC until mid-log phase and blocked with $10 \%$ bovine serum albumin. The lysate of grown rM.S was fractionated on $20 \%$ SDS-polyacrylamide gels and blotted onto nitrocellulose filters (Invitrogen; Thermo Fisher Scientific, Inc., Waltham, MA, USA). The membranes were blocked with $5 \%$ non-fat milk and incubated with a rabbit anti-human MAGEA3 antibody (Abgent, Inc., San Diego, CA, USA) at a dilution of 1:100 and a rabbit anti-human SSX2 antibody (Abcam, Cambridge, MA, USA) at a dilution of $1: 200$ or a mouse anti- $\beta$-actin monoclonal antibody at a dilution of 1:2,000 (Sigma-Aldrich; Merck KGaA, Darmstadt, Germany). The membranes were subsequently incubated with a goat anti-mouse or anti-rabbit horseradish peroxidase secondary antibody (Sigma-Aldrich; Merck KGaA). The protein complexes were detected using enhanced chemiluminescence reagents (Pierce; Thermo Fisher Scientific, Inc.).

The production of antibodies against MAGEA3 and SSX2 in the blood of immunized mice was determined using the purified MAGEA3 protein (Abnova, Walnut, CA, USA) or SSX2 protein (Abnova) separated by SDS-PAGE. All experiments were carried out at least three times. 
Immunization of mice. Seven-week-old and specific pathogen-free male BALB/c mice provided by the laboratory animal center of the Fourth Military Medical University were used for immunogenicity studies. All animal protocols were reviewed and approved by the Institutional Animal Care and Use Committee of the Fourth Military Medical University (ID11013). Mice were randomly divided into six groups (6 per group) to receive subcutaneous injections as follows: Normal control group (NC) received $0.2 \mathrm{ml}$ saline/mouse, $M$. smegmatis group infected with the $M$. smegmatis strain and received $1 \times 10^{6} \mathrm{CFU}$ empty pDE22 vector/mouse via the tail vein, recombinant $M$. smegmatis MAGEA3 (rM.S-M) infected with the $M$. smegmatis strain and transfected with pDE22-MAGEA3 at a dose of $1 \times 10^{6} \mathrm{CFU} /$ mouse, recombinant M. smegmatis SSX2 (rM.S-S) infected with the M. smegmatis strain and transfected with pDE22-SSX2 at a dose of $1 \times 10^{6} \mathrm{CFU} /$ mouse, recombinant $M$. smegmatis MAGEA3-SSX2 (rM.S-MS) infected with the M. smegmatis strain and transfected with pDE22-MAGEA3-SSX2 at a dose of $1 \times 10^{6} \mathrm{CFU} /$ mouse, recombinant $M$. smegmatis SSX2-MAGEA3 (rM.S-SM) infected with the M. smegmatis strain transfected with pDE22-SSX2-MAGEA3 at a dose of $1 \times 10^{6} \mathrm{CFU} /$ mouse. Mice were immunized once every 5 days with rM.S for a total of three times.

Immunotherapy in the tumor-bearing mice. Seven-week-old, specific pathogen-free male $\mathrm{BALB} / \mathrm{c}$ nude mice provided by the laboratory animal center of the Fourth Military Medical University were housed and monitored in a specific pathogen-free environment with sterile food and water in our animal facility. The human esophageal EC9706 cancer cell line, which was MAGEA3 and SSX2 double-positive cancer cell, was maintained in culture and prepared for injection as previously described (22). EC9706 tumor cells were cultured and inoculated subcutaneously into one site on the back surface of each BALB/c nude mouse at a concentration of $1 \times 10^{6}$ cells. Mice were cultured and observed until an obvious visible tumor appeared on the mouse back. Tumor-bearing mice were randomly divided into six groups with 6 mice each. The mice received the following different treatment: Normal control group (NC) receiving $100 \mu \mathrm{l} 0.9 \%$ saline/mouse, $M$. smegmatis group, rM.S-M group, rM.S-S group, rM.S-MS group and rM.S-SM group infected with the blood of the immunized mice from the same groups as the above via the tail vein at a dose of $100 \mu \mathrm{l} /$ mouse, respectively.

In the present study, all data collection was completed from 5 to 21 days after injection. The sizes of tumors were measured using a digital caliper in three dimensions $(\mathrm{L} \times \mathrm{W} \times \mathrm{H})$. The height of the tumors was determined by physically grasping the tumor by its base. Tumor volume was calculated using the following equation: Tumor Volume $=(\pi \cdot \mathrm{H}(\mathrm{H} 2+3 \cdot(\mathrm{L}+\mathrm{W} / 2))) / 6$. Mice were euthanized by inhalation of $\mathrm{CO}_{2}$ gas on day 21 of tumor growth. Tumors were dissected and weighed.

Statistical analysis. Data are presented as the mean \pm standard deviation (SD) from at least three independent experiments. Statistical analysis was perforned using SPSS16.0 (SPSS, Inc., Chicago, IL, USA). Student's t-test and $\chi^{2}$ test were used to analyze the difference between different groups. The

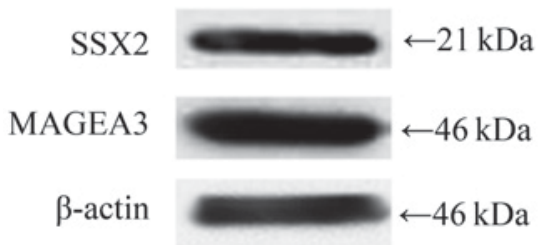

Figure 1. The protein expression of MAGEA3 and SSX2 in EC9706 cancer cells. Western blot assay was performed to detect the MAGEA3 and SSX2 protein expression in human esophageal EC9706 cancer cell line. $\beta$-actin is shown as a loading control. MAGEA3, melanoma-associated antigen A3.

comparison of multiple groups was carried out using one-way ANOVA followed by Tukey's post hoc test. $\mathrm{P}<0.05$ was considered to indicate a statistically significant difference.

\section{Results}

MAGEA3 and SSX2 were double-positive expressed in human esophageal EC9706 cancer cell line. Firstly, we detected the protein expression level of MAGEA3 and SSX2 in EC9706 cells, and the results showed that both MAGEA3 and SSX2 were highly expressed (Fig. 1).

Construction of rM.S strains and the expression of two fusion proteins. The optimized MAGEA3-SSX2 and SSX2-MAGEA3 fusion segments were synthesized in the pUC57 vector by Shanghai Generay Biotechnology Co., Ltd. (Shanghai, China) in BamHI and EcoRV restriction sites at the 5'and 3'ends. Then, the generated MAGEA3-SSX2 and SSX2-MAGEA3 fusion segments, along with the vector, were digested with PstI and ClaI restriction enzymes and ligated into pDE22 in the corresponding enzyme site at the 5'and 3' ends. Agarose gel electrophoresis of the PstI and ClaI digested plasmid showed the expected bands of $1,596 \mathrm{bp}$, representing the insert and pDE22, respectively (Fig. 2A). Also, sequencing showed that the target DNA was inserted correctly into the multi-cloning site of pDE22.

The pDE22-based constructs were electroporated into the fast-growing, non-pathogenic $M$. smegmatis strain to obtain a rM.S strain that could be easily manipulated in most laboratories. Following induction, MAGEA3-SSX2 and SSX2-MAGEA3 were expressed and corresponded to their predicted molecular masses of $67 \mathrm{kDa}$ (MAGEA3 approximately $46 \mathrm{kDa}$ and SSX2 approximately $21 \mathrm{kDa}$ ) on an SDS-PAGE gel (Fig. 2B). Western blotting showed that the MAGEA3-SSX2 and SSX2-MAGEA3 were recognized by the purchased rabbit anti-human SSX2 polyclonal antibody and anti-human MAGEA3 polyclonal antibody (Fig. 2C).

Antibody production in immunized mice. Mice were immunized once every 5 days with rM.S for a total of three times, as described in Methods. The expression of fusion protein-specific antibody levels after final vaccination is shown in Fig. 3. Immunized mice blood fusion protein-specific antibodies levels were detected by Western blot. Significantly specific specific antibodies levels were observed in mice of the groups vaccinated with the rM.S compared to the control group. In contrast, no specific antibodies were expressed between the control group and the M. smegmatis group. 
A

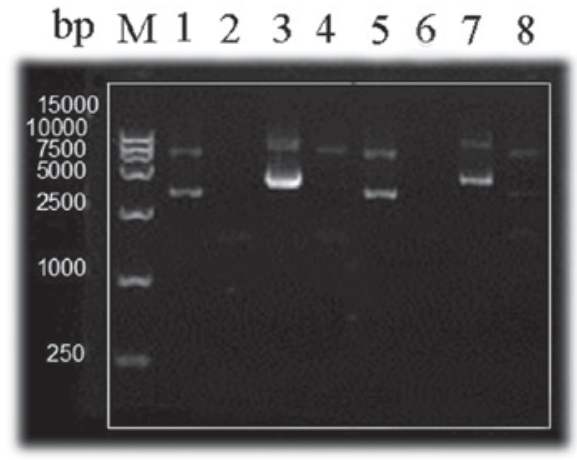

B $\begin{array}{lllllll}M & 1 & 2 & 3 & 4 & 5\end{array}$

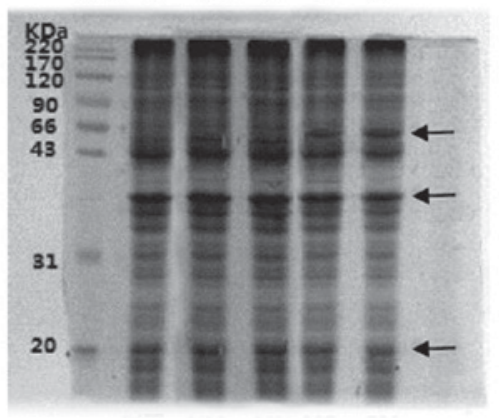

$\mathrm{C}$

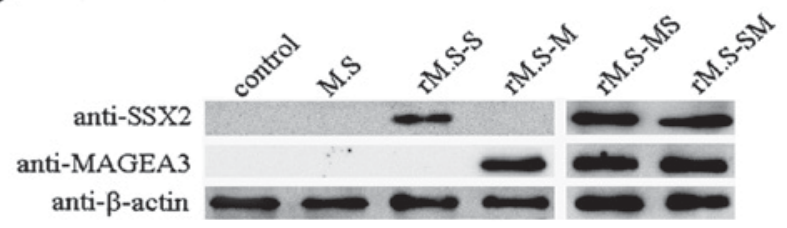

Figure 2. Identification of successfully constructed rM.S vaccine and detection the expression of MAGEA3-SSX2 (MS) and SSX2-MAGEA3 (SM) fusion proteins in the rM.S. (A) Agarose gel electrophoresis for the verification of recombinant pDE22 vector. Lane M, DNA marker; line 1, the empty vector of pDE22; line 2, rM.S; line 3, pDE22-MS; line 4, pDE22-MS digested with EcoRV and Cla1; line 5, the empty vector of pDE22; line 6, rM.S-SM; line 7, pDE22- SM; line 8, pDE22-SM digested with EcoRV and Cla1.(B) SDS-PAGE showed the expression of the MAGEA3 and SSX2 transgenes in the rM.S. Lane M, protein marker; line 1, M. smegmatis; line 2, MAGEA3-M. smegmatis; line 3, SSX2-M. smegmatis; line 4, MAGEA3-SSX2-rM.S; line 5, SSX2-MAGEA3-rM.S. MAGEA3-SSX2 and SSX2-MAGEA3 predicted molecular masses of $67 \mathrm{kDa}$ (MAGEA3 approximately $46 \mathrm{kDa}$ and SSX2 approximately $21 \mathrm{kDa})$. (C) Western blot analysis of MAGEA3 and SSX2 transgenes expression in the rM.S. MAGEA3, melanoma-associated antigen A3. rM.S, recombinant M. smegmatis; rM.S-MS, recombinant M. smegmatis MAGEA3-SSX2; rM.S-SM, recombinant M. smegmatis SSX2-MAGEA3; rM.S-S, recombinant M. smegmatis-SSX2; rM.S-M, recombinant M. smegmatis-MAGEA3.

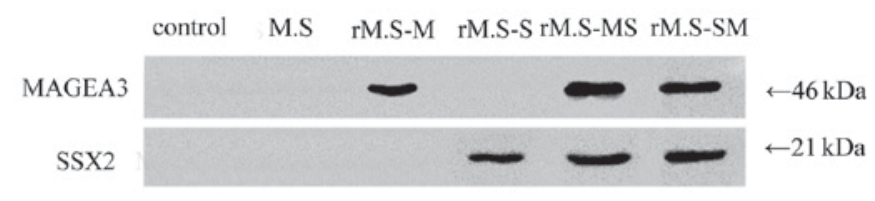

Figure 3. Western blot analysis of specific fusion protein antibody in immunized mouse blood. Mice were immunized once every 5 days with rM.S for a total of three times. The expression of blood fusion protein-specific antibody levels after final vaccination was determined using western blot analysis. MAGEA3, melanoma-associated antigen A3; rM.S, recombinant M. smegmatis; rM.S-MS, recombinant M. smegmatis MAGEA3-SSX2; rM.S-SM, recombinant M. smegmatis SSX2-MAGEA3; rM.S-S, recombinant $M$. smegmatis-SSX2; rM.S-M, recombinant $M$. smegmatis-MAGEA3.

The antitumor effect of the rM.S-MS and rM.S-SM was better than that of the single rM.S-M or rM.S-S. To establish the tumor-bearing mouse model, mice were injected using $1 \times 10^{6}$ EC9706 cells into one site of the ventral surface. Approximately 5 days later, the back surface of every mouse formed a macroscopic and palpable tumor. Following the detection of a palpable tumor, mice were treated daily by rM.S injection with $100 \mu \mathrm{l} /$ mouse or saline control. The rM.S-treated tumor volumes were lower compared with the control group (Fig. 4A). As shown in Fig. 3, rM.S-M and rM.S-S-stimulated mice group showed a slight decrease in tumor volume compared to the decrease in tumor volume of the rM.S-MS- or rM.S-SM-stimulated mice groups. There was no significant difference in tumor volume between rM.S-MS or rM.S-SM mice. This was accompanied by a significant reduction in the final tumor weight in the rM.S-treated mice. Tumors were dissected and weighed on day 21 of tumor growth (Fig. 4B). As shown in Fig. 3, thte rM.S-M- and rM.S-S-stimulated mice groups showed a slight decrease in tumor weight compared to the rM.S-MS- or rM.S-SM-stimulated mice groups. There was no significant difference in tumor weight between rM.S-MS or rM.S-SM mice. The result shows that MAGEA3 and SSX2 rM.S are better than the single MAGEA3 M. smegmatis or single SSX2 $M$. smegmatis for the demonstration of an antitumor effect. In addition, there was no significant difference in the antitumor effect of rM.S-MS or rM.S-SM.

\section{Discussion}

Mycobacterium smegmatis is a nonpathogenic species of the genus Mycobacterium which is easily manipulated to produce recombinant bacteria. Consequently, it is widely used as a live vaccine against cancer $(23,24)$. rM.S has been used to express various proteins in studies. A particular rM.S vaccine expressing a fusion protein containing ESAT- 6 and CFP10 induced higher humoral and cellular immunity than the M. bovis BCG vaccination in a mouse model (25). Similarly, Xu et al reported that fusion protein Ag85A-IL17A was expressed in rM.S vaccine which attenuated allergic airwat inflammation (26). Here, we demonstrated two kinds of novel vaccine vectors: rM.S-MS and rM.S-SM. rM.S-MS is a recombinant non-pathogenic M. smegmatis $\mathrm{MC}^{2} 155$ expressing the MAGEA3-SSX2 fusion protein; rM.S-SM is a rM.S expressing the SSX2-MAGEA3 fusion protein. Moreover, the M. smegmatis $\mathrm{MC}^{2} 155$ strain has been shown to be nonpathogenic following intravenous infections of SCID mice (27); we also verified the treatment effect 


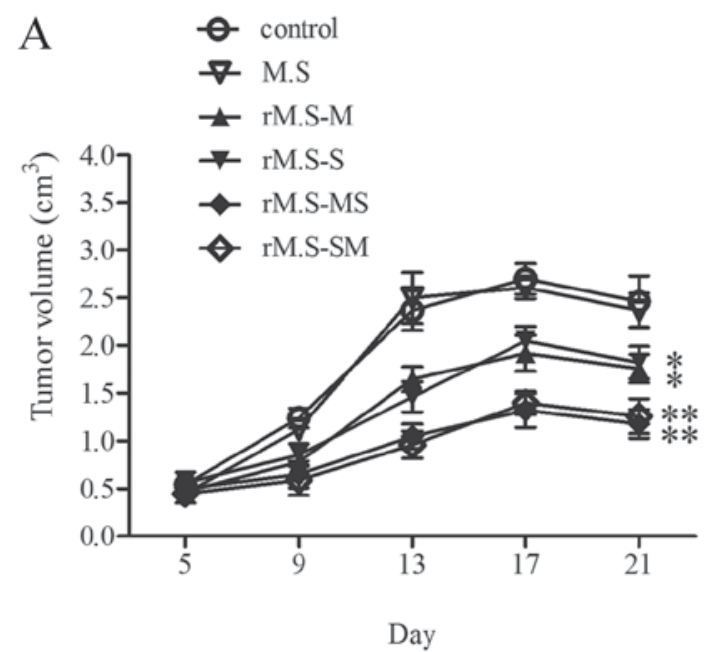

B

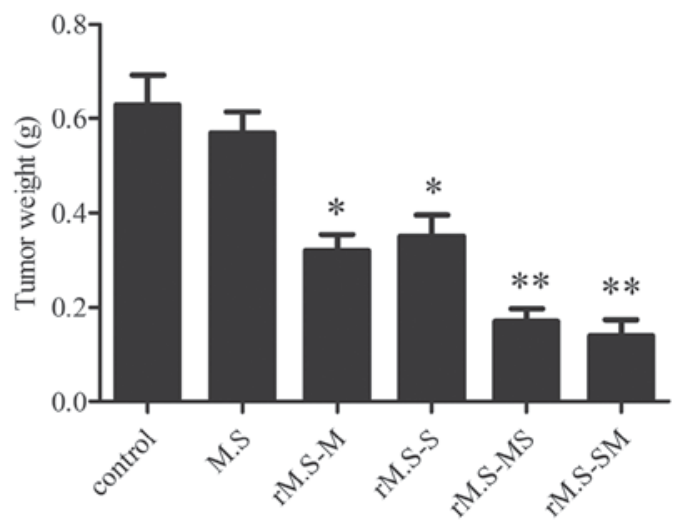

Figure 4. rM.S inhibits the esophageal tumor growth in an esophageal cancer mouse model. (A) Tumor volume was calculated for the 5 different time points and the rM.S treated tumor volumes were lower than those of the control group. (B) Tumors were dissected and weighed on day 21 of tumor growth. Data presented are mean \pm standard deviation. ${ }^{*} \mathrm{P}<0.05$ vs. control or MS; ${ }^{* *} \mathrm{P}<0.05$ vs. rM.S-M or rM.S-S. rM.S, recombinant $M$. smegmatis; rM.S-MS, recombinant M. smegmatis MAGEA3-SSX2; rM.S-SM, recombinant M. smegmatis SSX2-MAGEA3; rM.S-S, recombinant M. smegmatis-SSX2; rM.S-M, recombinant M. smegmatis-MAGEA3.

of tumors in the tumor-bearing mouse model through vaccine with recombinant strains.

We have shown that our recombinant vaccines rM.S-MS and rM.S-SM are capable of inducing a specific immune response in two different vaccination schemes (Fig. 2). This response probably reflects the immunogenicity of the recombinant fusion protein when used in this vaccine vector. The results of our group and others have confirmed that MAGEA3 and SSX2 tumor antigens with immunogenic and antigenic properties are represented by the epitopes expressed in the recombinant fusion protein (28-32). Both MAGEA3 and SSX2 belong to the group of cancer-testis antigens (CTA); CTA are immunogenic antigens with an expression that is largely restricted to testicular germ cells and a variety of malignancies, making them attractive targets for cancer immunotherapy $(11,33)$. The cancer-testis-X genes have been the principal targets of developing immunotherapies (33). MAGEA3 and SSX2 are all immunogenic CTA that have been shown to elicit coordinated humoral and cell-mediated immune responses $(33,34)$.

MAGEA3 is one of the best-characterized tumor antigens. Due to its tumor-restricted expression pattern and its recognition by both cytotoxic and helper $\mathrm{T}$ cells, it constitutes a promising tumor antigen for anticancer immunotherapy. Roeder et al demonstrated that MAGEA3 is a frequent tumor antigen of metastasized melanoma (29). Vansteenkiste et al demonstrated that MAGEA3 cancer immunotherapy is an active immunotherapy that has been evaluated in NSCLC (35). SSX gene products are expressed in tumors of different histological types and can be recognized by tumor-reactive CTLs from cancer patients. The immunogenicity of SSX-2 has been previously corroborated by detection of specific humoral and CD8+ $\mathrm{T}$ cell responses in cancer patients (36). Ayyoub et al also demonstrated that an SSX2-derived immunodominant $\mathrm{T}$ cell epitope is recognized by CD4+ T cells from melanoma patients in association with HLA-DR $(37,38)$. This is because MAGEA3 and SSX2 are able to trigger an immune response in the tumor. The aim of our study is to build a fusion protein composed of MAGEA3 and SSX2 and verify whether the fusion proteins' antitumor immune responses are enhanced by either of the two. In the present study, two fusion proteins were amplified in M. smegmatis. ELISA was used to detect whether anti-fusion protein antibodies are produced or not in vivo in immunized mice after the injection of rM.S vaccine. In Fig. 2, rM.S-MS group mice blood and the rM.S-SM group mice blood are able to produce both anti-MAGEA3 and anti-SSX2 antibodies. There was no significant difference in antibody concentration between the two groups. However, the antibody concentration of the rM.S-MS and rM.S-SM groups was significantly increased compared with the rM.S-M group and rM.S-S group, which only produce single protein antibodies. The results are in line with our expectations. Multiple-antigen combination tests may be more useful for the development of diagnostic antibody tests because of many antigens inducing serological responses.

The BALB/c mouse is the animal most commonly used as an in vivo model for $M$. smegmatis infection and constructed tumor-bearing mice model. The human esophageal EC9706 cancer cell line is a MAGEA3 and SSX2 double-positive tumor. In this study, BALB/c mice were immunized with rM.S-M, rM.S-S, rM.S-MS and rM.S-SM to provide experimental data to evaluate the effect of these proteins in M. smegmatis; blood was obtained containing the corresponding antibody to the treatment of tumor-bearing mice. According to our data after immunization, we extracted blood from the mice with the best antibody concentration for the following experiment. Treatment with the rM.S inhibits esophageal tumor growth in vivo in mice. The rM.S treated tumor volumes and weight were significantly reduced compared with the control group (Fig. 3).

In conclusion, in the current study we constructed rM.S vaccines, rM.S-MS and rM.S-SM, and demonstrated the immunogenicity of the vaccine. In addition, we demonstrated that the rM.S vaccine can activate the immune system and enhance 
the antitumor effect. The antitumor effect of the rM.S-MS and rM.S-SM is better than that of the single rM.S-M or rM.S-S.

\section{Acknowledgements}

The authors are grateful to Yinlan Bo's Laboratory, The Fourth Military Medical University, which made this work possible. The authors would also like to thank the clinicians and hospital staff who contributed to data collection for this study.

\section{Funding}

The present research received funding from the National Natural Science Foundation (grant nos. 31370926 and 81371774).

\section{Availability of data and materials}

All data generated or analyzed during this study are included in this published article.

\section{Authors' contributions}

WJ and $\mathrm{YX}$ conceived and designed the experiments. WJ, XL, JK, YL and YB performed the experiments. WJ and XL analyzed the data. WJ, XL and JK contributed reagents/materials/analysis tools. WJ and YX wrote the paper.

\section{Ethics approval and consent to participate}

All animal protocols were reviewed and approved by the Institutional Animal Care and Use Committee of the Fourth Military Medical University (ID11013).

\section{Patient consent for publication}

Not applicable.

\section{Competing interests}

The authors declare that they have no competing interests.

\section{References}

1. Lizée G, Cantu MA and Hwu P: Less yin, more yang: Confronting the barriers to cancer immunotherapy. Clin Cancer Res 13: 5250-5255, 2007.

2. Stewart TJ and Abrams SI: How tumours escape mass destruction. Oncogene 27: 5894-5903, 2008.

3. Stewart TJ and Smyth MJ: Improving cancer immunotherapy by targeting tumor-induced immune suppression. Cancer Metastasis Rev 30: 125-140, 2011.

4. Pierre-Audigier C, Jouanguy E, Lamhamedi S, Altare F, Rauzier J, Vincent V, Canioni D, Emile JF, Fischer A, Blanche S, et al: Fatal disseminated Mycobacterium smegmatis infection in a child with inherited interferon gamma receptor deficiency. Clin Infect Dis 24: 982-984, 1997.

5. Sweeney KA, Dao DN, Goldberg MF, Hsu T, Venkataswamy MM, Henao-Tamayo M, Ordway D, Sellers RS, Jain P, Chen B, et al: A recombinant Mycobacterium smegmatis induces potent bactericidal immunity against Mycobacterium tuberculosis. Nat Med 17: 1261-1268, 2011

6. van Faassen H, Dudani R, Krishnan L and Sad S: Prolonged antigen presentation, APC-, and CD8+ T cell turnover during mycobacterial infection: Comparison with Listeria monocytogenes. J Immunol 172: 3491-3500, 2004.
7. Cayabyab MJ, Hovav AH, Hsu T, Krivulka GR, Lifton MA, Gorgone DA, Fennelly GJ, Haynes BF, Jacobs WR Jr and Letvin NL: Generation of CD8+ T-cell responses by a recombinant nonpathogenic Mycobacterium smegmatis vaccine vector expressing human immunodeficiency virus type 1 Env. J Virol 80: 1645-1652, 2006.

8. Sang M, Lian Y, Zhou X and Shan B: MAGE-A family: Attractive targets for cancer immunotherapy. Vaccine 29: 8496-8500, 2011

9. Meek DW and Marcar L: MAGE-A antigens as targets in tumour therapy. Cancer Lett 324: 126-132, 2012.

10. Hofmann O, Caballero OL, Stevenson BJ, Chen YT, Cohen T, Chua R, Maher CA, Panji S, Schaefer U, Kruger A, et al: Genome-wide analysis of cancer/testis gene expression. Proc Natl Acad Sci USA 105: 20422-20427, 2008.

11. Scanlan MJ, Gure AO, Jungbluth AA, Old LJ and Chen YT: Cancer/testis antigens: An expanding family of targets for cancer immunotherapy. Immunol Rev 188: 22-32, 2002.

12. Gjerstorff MF, Andersen MH and Ditzel HJ: Oncogenic cancer/testis antigens: Prime candidates for immunotherapy. Oncotarget 6: 15772-15787, 2015.

13. Van den Eynde BJ and van der Bruggen P: T cell defined tumor antigens. Curr Opin Immunol 9: 684-693, 1997.

14. Gaugler B, Van den Eynde B, van der Bruggen P, Romero P, Gaforio JJ, De Plaen E, Lethé B, Brasseur F and Boon T: Human gene MAGE-3 codes for an antigen recognized on a melanoma by autologous cytolytic T lymphocytes. J Exp Med 179: 921-930, 1994.

15. Greve KB, Pøhl M, Olsen KE, Nielsen O, Ditzel HJ and Gjerstorff MF: SSX2-4 expression in early-stage non-small cell lung cancer. Tissue Antigens 83: 344-349, 2014.

16. Bloom JE and McNeel DG: SSX2 regulates focal adhesion but does not drive the epithelial to mesenchymal transition in prostate cancer. Oncotarget 7: 50997-51011, 2016.

17. Greve KB, Lindgreen JN, Terp MG, Pedersen CB, Schmidt S, Mollenhauer J, Kristensen SB, Andersen RS, Relster MM, Ditzel HJ and Gjerstorff MF: Ectopic expression of cancer/testis antigen SSX2 induces DNA damage and promotes genomic instability. Mol Oncol 9: 437-449, 2015.

18. Chen L, Zhou WB, Zhao Y, Liu XA, Ding Q, Zha XM and Wang S: Cancer/testis antigen SSX2 enhances invasiveness in MCF-7 cells by repressing ER $\alpha$ signaling. Int J Oncol 40: 1986-1994, 2012

19. Huang CJ, Chen RH, Vannelli T, Lee F, Ritter E, Ritter G, Old LJ and Batt CA: Expression and purification of the cancer antigen SSX2: A potential cancer vaccine. Protein Expr Purif 56: 212-219, 2007.

20. Türeci O, Sahin U, Schobert I, Koslowski M, Scmitt H, Schild HJ, Stenner F, Seitz G, Rammensee HG and Pfreundschuh M: The SSX-2 gene, which is involved in the $\mathrm{t}(\mathrm{X} ; 18)$ translocation of synovial sarcomas, codes for the human tumor antigen HOM-MEL-40. Cancer Res 56: 4766-4772, 1996.

21. Bardarov S, Kriakov J, Carriere C, Yu S, Vaamonde C, McAdam RA, Bloom BR, Hatfull GF and Jacobs WR Jr: Conditionally replicating mycobacteriophages: A system for transposon delivery to Mycobacterium tuberculosis. Proc Natl Acad Sci USA 94: 10961-10966, 1997.

22. Xu H, Crawford D, Hutchinson KR, Youtz DJ, Lucchesi PA, Velten M, McCarthy DO and Wold LE: Myocardial dysfunction in an animal model of cancer cachexia. Life Sci 88: 406-410, 2011.

23. Young SL, Murphy M, Zhu XW, Harnden P, O'Donnell MA, James K, Patel PM, Selby PJ and Jackson AM: Cytokine-modified Mycobacterium smegmatis as a novel anticancer immunotherapy. Int J Cancer 112: 653-660, 2004.

24. Luo Y, Henning J and O'Donnell MA: Th1 cytokine-secreting recombinant Mycobacterium bovis bacillus Calmette-Guérin and prospective use in immunotherapy of bladder cancer. Clin Dev Immunol 2011: 728930, 2011.

25. Zhang H, Peng P, Miao S, Zhao Y, Mao F, Wang L, Bai Y, Xu Z, Wei S and Shi C: Recombinant Mycobacterium smegmatis expressing an ESAT6-CFP10 fusion protein induces anti-mycobacterial immune responses and protects against Mycobacterium tuberculosis challenge in mice. Scand J Immunol 72: 349-357, 2010.

26. Xu W, Chen L, Guo S, Wu L and Zhang J: Intranasal administration of recombinant Mycobacterium smegmatis inducing IL-17A autoantibody attenuates airway inflammation in a murine model of allergic asthma. PLoS One 11: e0151581, 2016. 
27. Bange FC, Collins FM and Jacobs WR Jr: Survival of mice infected with Mycobacterium smegmatis containing large DNA fragments from Mycobacterium tuberculosis. Tuber Lung Dis 79: 171-180, 1999

28. Kirkin AF, Dzhandzhugazyan KN and Zeuthen J: Cancer/testis antigens: Structural and immunobiological properties. Cancer Invest 20: 222-236, 2002.

29. Roeder C, Schuler-Thurner B, Berchtold S, Vieth G, Driesch Pv, Schuler G and Lüftl M: MAGE-A3 is a frequent tumor antigen of metastasized melanoma. Arch Dermatol Res 296: 314-319, 2005.

30. Moeller I, Spagnoli GC, Finke J, Veelken H and Houet L: Uptake routes of tumor-antigen MAGE-A3 by dendritic cells determine priming of naïve T-cell subtypes. Cancer Immunol Immunother 61: 2079-2090, 2012.

31. Valmori D, Qian F, Ayyoub M, Renner C, Merlo A, Gnjatic S, Stockert E, Driscoll D, Lele S, Old LJ and Odunsi K: Expression of synovial sarcoma X (SSX) antigens in epithelial ovarian cancer and identification of SSX-4 epitopes recognized by CD4+ T cells. Clin Cancer Res 12: 398-404, 2006.

32. Abate-Daga D, Speiser DE, Chinnasamy N, Zheng Z, Xu H, Feldman SA, Rosenberg SA and Morgan RA: Development of a $\mathrm{T}$ cell receptor targeting an HLA-A*0201 restricted epitope from the cancer-testis antigen SSX2 for adoptive immunotherapy of cancer. PLoS One 9: e93321, 2014

33. Caballero OL and Chen YT: Cancer/testis (CT) antigens: Potential targets for immunotherapy. Cancer Sci 100: 2014-2021, 2009.
34. Hemminger JA, Toland AE, Scharschmidt TJ, Mayerson JL, Guttridge DC and Iwenofu OH: Expression of cancer-testis antigens MAGEA1, MAGEA3, ACRBP, PRAME, SSX2, and CTAG2 in myxoid and round cell liposarcoma. Mod Pathol 27: 1238-1245, 2014.

35. Vansteenkiste J,Zielinski M, Linder A, Dahabreh J, Gonzalez EE, Malinowski W, Lopez-Brea M, Vanakesa T, Jassem J, Kalofonos H, et al: Adjuvant MAGE-A3 immunotherapy in resected non-small-cell lung cancer: Phase II randomized study results. J Clin Oncol 31: 2396-2403, 2013.

36. Bricard G, Bouzourene H, Martinet O, Rimoldi D, Halkic N, Gillet M, Chaubert P, Macdonald HR, Romero P, Cerottini JC and Speiser DE: Naturally acquired MAGE-A10- and SSX-2-specific CD8+ T cell responses in patients with hepatocellular carcinoma. J Immunol 174: 1709-1716, 2005.

37. Ayyoub M, Hesdorffer CS, Metthez G, Stevanovic S, Ritter G, Chen YT, Old LJ, Speiser D, Cerottini JC and Valmori D: Identification of an SSX-2 epitope presented by dendritic cells to circulating autologous CD4+ T cells. J Immunol 172: 7206-7211, 2004.

38. Ayyoub M, Stevanovic S, Sahin U, Guillaume $P$, Servis C, Rimoldi D, Valmori D, Romero P, Cerottini JC, Rammensee HG, et al: Proteasome-assisted identification of a SSX-2-derived epitope recognized by tumor-reactive CTL infiltrating metastatic melanoma. J Immunol 168: 1717-1722, 2002. 